\title{
$C_{0} \mathbf{I} \cdot \mathbf{R}^{\circ} \mathbf{P} \cdot \mathrm{E}^{\prime} \mathrm{E}$
}

Centre Interuniversitaire sur le Risque, les Politiques Économiques et l'Emploi

Cahier de recherche/Working Paper 13-28

\section{Implied Expected Returns and the Choice of a Mean-Variance Efficient Portfolio Proxy}

David Ardia

Kris Boudt

Septembre/September 2013

Ardia : Corresponding author. Département de finance, assurance et immobilier, Université Laval, Québec City (Québec), Canada; and CIRPÉE

david.ardia@fsa.ulaval.ca

Boudt : Solvay Business School, Vrije Universiteit Brussel; and Faculty of Economics and Business, VU University Amsterdam, The Netherlands

kris.boudt@vub.ac.be

Work in progress; comments are welcome. We are grateful to Enrico Schumann, Piet Sercu, Marjan Wauters and participants at the IAF seminar (University of Neuchâtel 2013), the R/Finance 2013 and the SSES 2013 conferences for useful suggestions. Any remaining errors or shortcomings are the authors' responsibility. 


\begin{abstract}
:
We propose to compute the implied expected returns from several candidate meanvariance efficient portfolios, exploiting the fundamental relation between the expected returns, covariance matrix and the corresponding set of mean-variance efficient portfolios. Over the 1987-2012 period and for the universe of S\&P 100 stocks, we find that a mean-variance efficient investor would have been willing to pay between a $1.7 \%$ and $4.2 \%$ management fee to switch from mean-variance investing using implied expected returns from the market capitalization weighted portfolio to mean-variance investing using the implied expected returns from the equal-risk-contribution portfolio.
\end{abstract}

Keywords: Implied expected return, mean-variance, model selection, portfolio allocation, reverse engineering, risk-based allocation

JEL Classification: G11, G12, C13, C31 


\section{Introduction}

A growing amount of capital is attracted by so-called "smart beta" equity solutions, such as the equal-weighted, equal-risk-contribution and maximum diversification portfolio. ${ }^{1}$ The determination of their portfolio weights does not require any return forecast, but, from the portfolio weight and the covariance matrix, an implied expected return can be computed. We investigate the usefulness of this alternative implied expected return for mean-variance portfolio investors, in comparison with the standard approach of deriving implied expected returns from the market capitalization weighted portfolio.

We start from three fundamental insights. First, if $\mathbf{w}$ is a mean-variance efficient portfolio with respect to a universe of assets with known expected returns vector $\boldsymbol{\mu}$ and covariance matrix $\boldsymbol{\Sigma}$, then there exists a linear relation between $\boldsymbol{\mu}$ and $\Sigma \mathrm{w}$. Second, covariances can be much more accurately estimated than expected returns. ${ }^{2}$ Hence, if a mean-variance efficient weight vector is known and a covariance matrix has been accurately estimated, the above-mentioned linear relation can be exploited to create implied expected returns which have a higher accuracy than sample means. The idea of reverse engineering for expected returns was first advocated by Best and Grauer (1985), and almost always applied with the market capitalization portfolio, which is well known to be mean-variance efficient under the Capital Asset Pricing Model (CAPM) assumptions. Third, in realistic markets, stock prices may deviate from their fundamental values and market capitalization portfolios tend to invest disproportionately in stocks that are overpriced and underinvest in stocks that are under-priced (Arnott et al., 2005; Treynor, 2005). Hence, we advocate that the market capitalization weighted portfolio is only one possible proxy for a mean-variance efficient portfolio, and that other proxies may lead to more accurate expected returns.

Our main hypothesis is that there exist other (expected) return-insensitive proxies for meanvariance efficient portfolio weights that lead to more accurate expected return predictions. In particular, we will test this hypothesis for the smoothed market capitalization portfolio (Chen et al., 2007), the equally-weighted portfolio (DeMiguel et al., 2009), the equal-risk-contribution portfolio (Maillard et al., 2010), the maximum diversification portfolio (Choueifaty and Coignard, 2008) and the risk-efficient portfolio (Amenc et al., 2011). See e.g. Lee (2011) for a recent overview.

To our knowledge, this paper is the first to test the sensitivity of implied expected returns to the choice of mean-variance efficient portfolio. Over the 1987-2012 period and for the universe of S\&P 100 stocks, we find a substantial improvement in the performance of mean-variance efficient

\footnotetext{
${ }^{1}$ According to Flood (2013) global assets held in advanced beta strategies grew to 142 billion USD at the end of March 2013, up from just 58 billion USD at the end of 2010.

${ }^{2}$ We restrict our attention to time series methods that extrapolate observed return dynamics into the future. The predictability of asset returns using state variables such as dividend-price or dividend-earnings ratios is a matter of controversy in the literature and beyond the scope of this paper. See e.g. Campbel and Thompson (2007) and Welch and Goyal (2007). There are at least two explanations for why expected returns are more difficult to forecast than covariance matrices using time series econometrics. The first one is that, the mean and covariance matrix are time-varying parameters. Since the time-variation in the covariance matrix is much more persistent than the timevariation in the expected return, covariance matrices can me more accurately predicted than mean returns. Second, as it is commonly done in practice, we will use weekly observations to estimate the covariance matrix over a monthly horizon. As shown by Merton (1980), using higher sampling frequencies improves significantly the accuracy of the covariance estimator, but not the estimation of the mean parameter.
} 
portfolios computed using the implied expected returns from the equal-risk-contribution portfolio, compared with the standard use of implied expected returns from the market capitalization weighted portfolio. This result is robust with respect to the choice of the level of risk aversion as well as the type of gross exposure constraint (no short sales versus 130-30 allocation rule) implemented in the portfolio.

The remainder of the paper is organized as follows. Section 2 presents the methodology to compute implied expected return from mean-variance efficient portfolios. The different proxies that we consider are presented in Section 3. Section 4 compares the empirical differences in implied expected returns. The portfolio allocation methods, the choice of mean-variance efficient proxy and the sensitivity of the portfolios results are discussed in Section 5. Section 6 concludes.

\section{Implied expected return methodology}

We consider a market with $N$ risky securities and denote a generic portfolio in this market by the $(N \times 1)$ vector $\mathbf{w}$. The expected arithmetic returns (in excess of the risk-free rate) at the desired holding horizon are denoted by the $(N \times 1)$ vector $\boldsymbol{\mu}$ and the corresponding $(N \times N)$ covariance matrix of arithmetic returns is denoted by $\Sigma$. We drop time dimension for simplicity but will consider them as time-dependent. We denote by $\iota$ the $(N \times 1)$ vector of ones and by 0 the $(N \times 1)$ vector of zeros.

Our analysis builds on the assumption of mean-variance preferences. Let $0<\gamma<\infty$ be the risk aversion parameter. The mean-variance optimization problem is:

$$
\mathbf{w}^{*} \equiv \underset{\mathbf{w} \in \mathcal{C}_{\mathrm{FI}}}{\operatorname{argmax}}\left\{\boldsymbol{\mu}^{\prime} \mathbf{w}-\frac{1}{2} \gamma \mathbf{w}^{\prime} \mathbf{\Sigma} \mathbf{w}\right\}
$$

where $\mathcal{C}_{\mathrm{FI}} \equiv\left\{\mathbf{w} \in \mathbb{R}^{N} \mid \mathbf{w}^{\prime} \iota=1\right\}$ is the full-investment constraint. The Lagrangian corresponding to (1) is:

$$
\mathcal{L}(\mathbf{w}, l) \equiv \mathbf{w}^{\prime} \boldsymbol{\mu}-\frac{\gamma}{2} \mathbf{w}^{\prime} \mathbf{\Sigma} \mathbf{w}-l\left(\mathbf{w}^{\prime} \iota-1\right)
$$

with $l \in \mathbb{R}$. The corresponding first order conditions are:

$$
\begin{aligned}
\boldsymbol{\mu}-\gamma \boldsymbol{\Sigma} \mathbf{w}-l \iota & =\mathbf{0} \\
\mathbf{w}^{\prime} \iota & =1 .
\end{aligned}
$$

From (3), we note the linear relationship between $\boldsymbol{\mu}$ and $\Sigma \mathrm{w}$ :

$$
\boldsymbol{\mu}=l \iota+\gamma \boldsymbol{\Sigma} \mathbf{w}
$$

Note that as $\gamma$ is finite, (5) excludes the minimum variance portfolio.

The linear relationship in (5) is well known and has been used to compute the so-called implied expected returns, using the market capitalization weighted portfolio as a proxy for a meanvariance efficient portfolio. One of the first comprehensive treatments of this approach is Best and Grauer (1985). The practical application of implied expected returns was boosted by the research 
of Black and Litterman (1992). The latter show that, under the additional assumptions of the CAPM, the market implied expected returns can be seen as the neutral equilibrium expected returns, i.e., the levels of expected returns such that all investors are satisfied holding the outstanding asset weights. Recently, Levy and Roll (2010) propose to shrink the sample mean and covariance estimates towards levels that are compatible with the linear relationship in (1) and found that, for the market capitalization weighted portfolio, only relatively small changes are needed to achieve this. Interestingly, Ni et al. (2011) show that using those improved expected returns and covariance estimates, better out-of-sample performance of portfolios can be achieved. As in previous research, our goal is to obtain a more accurate expected returns' forecast, but we advocate that the market capitalization weighted portfolio is only one possible proxy for a mean-variance efficient portfolio, and that other proxies may lead to more accurate implied expected returns.

The linear relationship in (5) holds for variables that are not directly observable, namely the mean and covariance matrix of the returns and the mean-variance efficient weights vector, as well as the intercept and slope parameters $l$ and $\gamma$. We assume that there exist (possibly noisy) proxies for $\boldsymbol{\mu}, \boldsymbol{\Sigma}$ and $\mathrm{w}$, denoted by $\hat{\boldsymbol{\mu}}, \hat{\boldsymbol{\Sigma}}$ and $\hat{\mathrm{w}}$, and estimate the remaining intercept and slope parameters as the values for which the regression fit is as close as possible (in terms of mean squared error) to $\hat{\boldsymbol{\mu}}$, while satisfying the equilibrium condition in (5):

$$
y_{i}=a+b x_{i}+\varepsilon_{i},
$$

where $y_{i} \equiv \hat{\mu}_{i}$ and $x_{i} \equiv[\hat{\boldsymbol{\Sigma}} \hat{\mathbf{w}}]_{i}, \varepsilon_{i}$ is an error term and $a$ and $b$ are the least squares regression parameters under the restriction that $b>0$ as it estimates the risk aversion parameter $\gamma$ :

$$
\begin{aligned}
& \bar{y} \equiv \frac{1}{N} \sum_{i=1}^{N} y_{i} \\
& \bar{x} \equiv \frac{1}{N} \sum_{i=1}^{N} x_{i} \\
& b \equiv \max \left\{\frac{\sum_{i=1}^{N}\left(x_{i}-\bar{x}\right)\left(y_{i}-\bar{y}\right)}{\sum_{i=1}^{N}\left(x_{i}-\bar{x}\right)^{2}}, 0\right\} \\
& a \equiv \bar{y}-b \bar{x} .
\end{aligned}
$$

Alternatively, $a$ and $b$ can be estimated by generalized least squares. In the same spirit as Levy and Roll (2010) one could then set the weight matrix as the diagonal matrix of which the elements equal the inverse of the stocks' variance.

The implied expected return estimate $\tilde{\boldsymbol{\mu}}$ is given by the fit of the regression in (6):

$$
\tilde{\boldsymbol{\mu}} \equiv a+b(\hat{\boldsymbol{\Sigma}} \hat{\mathbf{w}}) .
$$

The implied expected return estimates $\tilde{\boldsymbol{\mu}}$ can thus be interpreted as the first-step mean estimate $\hat{\boldsymbol{\mu}}$ (typically, the historical average return on rolling samples) that has been adjusted such that it respects the assumption of mean-variance efficiency of the reference portfolio. 
Finally, we wish to note that the implied expected return estimator in (6) has two important features in common with the Bayes-Stein estimator of Jorion (1986). First, it does not estimate the expected return of each risky asset separately (in which case the sample mean would be optimal), but through the use of the covariance matrix, the mean-variance efficient proxy and the use of the cross-sectional sum of squared errors as loss function, it accounts for the information contained in the other series. Second, the less informative $\hat{\Sigma} \hat{\mathbf{w}}$ in (6) is for the first step mean estimate $\tilde{\mu}$, the more the implied return estimates will be "shrunk" toward the grand mean. In the special case where $b=0$, the implied expected return is the same for all risky assets and coincides with the cross-sectional average of the first-step mean estimate $\hat{\boldsymbol{\mu}}$.

\section{Proxies for mean-variance efficient portfolios}

Since the seminal work by Best and Grauer (1985) and Black and Litterman (1992), it has become standard to use the market capitalization portfolio for computing implied expected returns. The motivation for this is that, under the CAPM assumptions, the market portfolio, which we denote by $\mathbf{w}_{\mathrm{mkt}}$, has the mean-variance efficiency property. An increasing body of literature has criticized the mean-variance efficiency of the market capitalization weighted portfolio, and proposed alternatives that (under different assumptions) are mean-variance efficient.

The first strand of research that we consider is the fundamental indexing literature (Arnott et al., 2005; Treynor, 2005) arguing that in noisy markets, overpriced stocks receive a higher weight in the market capitalization weighted portfolio than the efficient one, and vice versa for under-priced stocks. If pricing errors are corrected, this leads to a drag on returns for market capitalization based portfolios. Chen et al. (2007) show that by smoothing the market cap weights, pricing errors are reduced and hence a more efficient estimate of the fundamental market capitalization weights are obtained. We take the same setup than in Chen et al. (2007) and consider the median capitalization weights over a five weeks period. We denote this portfolio by $\mathbf{w}_{\text {smkt }}$.

A second strand of research relates to the general objective of achieving diversified portfolios, whereby the focus of diversification implicitly leads to a portfolio that is mean-variance efficient under some assumptions.

- Equally-weighted portfolio:

DeMiguel et al. (2009) show that the $1 / N$ allocation rule outperforms several optimized portfolios. This portfolio is mean-variance efficient when the expected returns $\boldsymbol{\mu}$ are proportional to the total risk $\Sigma \iota$. In fact, the generic solution to the mean-variance optimization problem in (1) is:

$$
\mathbf{w}=\frac{1}{\gamma} \boldsymbol{\Sigma}^{-1} \boldsymbol{\mu} .
$$

Replacing $\boldsymbol{\mu}$ with a multiple of $\boldsymbol{\Sigma} \iota$ leads to the equally-weighted portfolio. We denote this portfolio by $\mathbf{w}_{\mathrm{ew}}$. 
- Equal-risk-contribution portfolio:

For a portfolio w, the percentage volatility risk contribution of the $i$ th asset in the portfolio is given by:

$$
\% R C_{i} \equiv \frac{w_{i}[\mathbf{\Sigma} \mathbf{w}]_{i}}{\mathbf{w}^{\prime} \mathbf{\Sigma} \mathbf{w}}
$$

The equal-risk-contribution portfolio is the portfolio for which all assets contribute equally to the overall risk of the portfolio. Numerically, it is computed by solving the following optimization problem ${ }^{3}$ :

$$
\mathbf{w}_{\mathrm{erc}} \equiv \underset{\mathbf{w} \in \mathcal{C}_{\mathrm{FI}}}{\operatorname{argmin}}\left\{\sum_{i=1}^{N}\left(\% R C_{i}-\frac{1}{N}\right)^{2}\right\},
$$

with $\mathcal{C}_{\mathrm{FI}} \equiv\left\{\mathbf{w} \in \mathbb{R}^{N} \mid \mathbf{w}^{\prime} \iota=1\right\}$, the set of weights guaranteeing that the portfolio is fully invested.

Maillard et al. (2010) derive the theoretical properties of the equal-risk-contribution portfolio and show that its volatility is located between those of the minimum variance and the equally-weighted portfolio. From the equal-risk-contribution constraint that $\% R C_{i}=1 / N$ (for all $i=1, \ldots, N$ ) and the generic solution for mean-variance efficient portfolios (1), it follows that the equal-risk-contribution portfolio is mean-variance efficient when:

$$
\boldsymbol{\mu}=\frac{\gamma}{N}\left(\mathbf{w}^{\prime} \boldsymbol{\Sigma} \mathbf{w}\right) \boldsymbol{\Sigma}(\boldsymbol{\iota} \cdot /(\boldsymbol{\Sigma} \mathbf{w})
$$

where ./ stands for element-wise division.

- Maximum diversification portfolio:

Let $\boldsymbol{\sigma} \equiv \sqrt{\operatorname{diag}(\boldsymbol{\Sigma})}$ be the $(N \times 1)$ vector of standard deviations of arithmetic returns of the $N$ assets in the universe. An important property of the portfolio standard deviation is its sub-additivity, meaning that, because of diversification effects, the portfolio's standard deviation is always less or equal to the weighted average volatility:

$$
\sqrt{\mathbf{w}^{\prime} \Sigma \mathbf{w}} \leq \mathbf{w}^{\prime} \sigma
$$

or equivalently that the ratio between the weighted average volatility and the portfolio volatility exceeds one:

$$
D R(\mathbf{w}) \equiv \frac{\mathbf{w}^{\prime} \boldsymbol{\sigma}}{\sqrt{\mathbf{w}^{\prime} \mathbf{\Sigma} \mathbf{w}}} \geq 1
$$

\footnotetext{
${ }^{3}$ If a numerical solution is not found, we follow the recommendation in Maillard et al. (2010) and slightly modify the problem and optimize over the $N$-dimensional vector $\mathbf{u}$ such that $\mathbf{w} \equiv \mathbf{u} /\left(\mathbf{u}^{\prime} \iota\right)$, under the constraint that $\mathbf{u} \geq \mathbf{0}$ and $\mathbf{u}^{\prime} \iota>0$. This new optimization problem is easier to solve numerically as an inequality constraint is less restrictive than the full investment equality constraint.
} 
Choueifaty and Coignard (2008) call this the portfolio's diversification ratio and define the maximum diversification portfolio as the portfolio that has the highest diversification ratio. Choueifaty et al. (2011) show that, in the special case where returns are proportional to their volatility, the maximum diversification portfolio coincides with the maximum Sharpe ratio portfolio. This special case is also discussed by Martellini (2008). We denote this portfolio by $\mathbf{w}_{\mathrm{md}}$.

- Risk-efficient portfolio:

Amenc et al. (2011) recommend to construct a maximum Sharpe portfolio under the assumption that the stock's expected return is a deterministic function of its semi-deviation and the cross-sectional distribution of semi-deviations, and where portfolio weights are constrained to be between $1 /(2 N)$ and $1 / N$. More precisely, they sort stocks by their semi-deviation, form decile portfolios and then compute the median semi-deviation of stocks in each decile portfolio which we denote by $\xi_{j}(j=1, \ldots, 10)$. The so-called risk-efficient portfolio is then given by:

$$
\mathbf{w}_{\text {ref }} \equiv \underset{\mathbf{w} \in \mathcal{C}_{\mathrm{FI}^{*}}}{\operatorname{argmax}}\left\{\frac{\mathbf{w}^{\prime} \boldsymbol{J} \boldsymbol{\xi}}{\sqrt{\mathbf{w}^{\prime} \boldsymbol{\Sigma} \mathbf{w}}}\right\},
$$

where $\boldsymbol{J}$ is a $(N \times 10)$ matrix of zeros whose $(i, j)$-th element is one if the semi-deviation of stock $i$ belongs to decile $j, \boldsymbol{\xi} \equiv\left(\xi_{1}, \ldots, \xi_{10}\right)^{\prime}$ and $\mathcal{C}_{\mathrm{FI}^{*}} \equiv\left\{\mathbf{w} \in \mathbb{R}^{N} \mid \mathbf{w}^{\prime} \iota=1,(1 / 2 N) \iota \leq\right.$ $\mathbf{w} \leq(1 / N) \boldsymbol{\iota}\}$ is the full-investment constraint with bound restrictions on the portfolio weights.

Overall, besides the mean forecast obtained by extrapolating past returns, we thus have six implied expected returns methods we will investigate. In what follows, we refer to the six implied expected returns methods by ir-mkt, ir-smkt, ir-ew, ir-erc, ir-md and ir-ref.

\section{Implied expected returns for $S \& P 100$ constituents}

In the next section, we will investigate the economic value of implied expected returns from alternative risk-based portfolios for an investor with mean-variance utility function and investing in the universe of hundred end-of-month largest S\&P 500 equities. The investor rebalances his portfolio on a monthly basis. This section introduces our dataset used to analyze the sensitivity of implied expected returns to the choice of proxy and provides descriptive statistics on the crosssectional and time series variation of the different sets of implied expected returns.

\subsection{Data}

Our sample period ranges from January 1984 to December 2012, and the estimation of the mean and covariance is performed using a rolling window of three years (156 weeks), which is common practice in the financial industry. For the first-step estimate of the expected return $\hat{\boldsymbol{\mu}}$ we use the average of monthly arithmetic returns. For the covariance matrix estimator $\hat{\Sigma}$, we use weekly log-returns and an exponential smoothing estimate with a decay parameter of 0.94 as 
advocated by RiskMetrics (RiskMetrics Group, 1996). The covariance matrix is initialized with the shrinkage approach of Ledoit and Wolf (2003). The covariance matrix is then projected and converted into a monthly arithmetic returns covariance matrix estimator as in Meucci $(2001,2004)$.

We will assume end-of-month rebalancing and restrict the cross-section to the universe of the hundred end-of-month largest S\&P 500 equities for which there are no missing observations in the estimation sample used to forecast the covariance matrix and expected returns. For convenience we will call this henceforth the S\&P 100 universe. The evaluation period thus ranges from January 1987 to December 2012, for a total of 312 monthly observations. The data have been retrieved from COMPUSTAT. In addition to the adjusted prices, we collected the market capitalization at the end of each month. The risk-free rate is the three-month Treasury bill. All figures are in USD.

\subsection{Differences in implied expected returns}

Before proceeding to the economic analysis through portfolio analysis, we first use descriptive statistics to analyze the difference in implied expected returns. We report in Table 1 the Pearson and Spearman correlations between the implied expected returns of the various methods. They have been estimated using 31,200 observations (312 rebalancing periods for 100 stocks). We find that the correlation between the implied expected returns obtained using different benchmarks is always higher than $90 \%$, while the correlation between the implied return and the returns obtained using the rolling three-year sample average estimator is around $65 \%$. The correlation between the implied expected returns obtained using the market weights, smoothed market weights and equal weights is extremely high (above 99\%).

\begin{tabular}{lccccccc}
\hline & $\tilde{\boldsymbol{\mu}}_{\text {ir-mkt }}$ & $\tilde{\boldsymbol{\mu}}_{\text {ir-smkt }}$ & $\tilde{\boldsymbol{\mu}}_{\text {ir-ew }}$ & $\tilde{\boldsymbol{\mu}}_{\text {ir-erc }}$ & $\tilde{\boldsymbol{\mu}}_{\text {ir-md }}$ & $\tilde{\boldsymbol{\mu}}_{\text {ir-ref }}$ & $\hat{\boldsymbol{\mu}}_{\text {rs }}$ \\
\hline$\tilde{\boldsymbol{\mu}}_{\text {ir-mkt }}$ & & 0.998 & 0.994 & 0.951 & 0.943 & 0.984 & 0.669 \\
$\tilde{\boldsymbol{\mu}}_{\text {ir-smkt }}$ & 0.998 & & 0.993 & 0.951 & 0.941 & 0.980 & 0.663 \\
$\tilde{\boldsymbol{\mu}}_{\text {ir-ew }}$ & 0.994 & 0.992 & & 0.960 & 0.950 & 0.990 & 0.664 \\
$\tilde{\boldsymbol{\mu}}_{\text {ir-erc }}$ & 0.969 & 0.968 & 0.975 & & 0.939 & 0.964 & 0.630 \\
$\tilde{\boldsymbol{\mu}}_{\text {ir-md }}$ & 0.950 & 0.948 & 0.956 & 0.953 & & 0.966 & 0.650 \\
$\tilde{\boldsymbol{\mu}}_{\text {ir-ref }}$ & 0.985 & 0.982 & 0.991 & 0.977 & 0.970 & & 0.665 \\
$\hat{\boldsymbol{\mu}}_{\text {rs }}$ & 0.623 & 0.620 & 0.626 & 0.617 & 0.622 & 0.628 & \\
\hline
\end{tabular}

Table 1: Correlation matrix between the implied expected returns of the various methods. Upper part reports the standard correlations while the lower part reports Spearman's rank correlations. $\tilde{\mu}_{\text {ir-mkt }}$ : implied expected returns with the market capitalization portfolio; $\tilde{\mu}_{\text {ir-smkt }}:$ smoothed market portfolio; $\tilde{\mu}_{\text {ir-ew }}$ : equally-weighted portfolio; $\tilde{\mu}_{\text {ir-erc }}$ : equal-risk-contribution portfolio; $\tilde{\boldsymbol{\mu}}_{\text {ir-md }}:$ maximum diversification portfolio; $\tilde{\boldsymbol{\mu}}_{\text {ir-ref }}:$ risk-efficient portfolio; $\hat{\boldsymbol{\mu}}_{\mathrm{rs}}:$ threeyear rolling sample average. The evaluation period ranges from January 1987 to December 2012 for a total of 312 monthly observations.

In Figure 1 we zoom-in on the relation between implied expected returns and the rolling sample estimator through a scatter plot diagram. To save space, we do not report the scatter plots for the implied expected returns computed using the smoothed market weighted and equally weighted benchmark, but they are similar to the scatter plot for implied expected returns from the market capitalization weighted portfolio. We immediately see the reduction of the dispersion in the implied expected returns forecasts compared with the rolling sample estimates of the implied 
expected returns. The standard deviation of the rolling sample estimator is 1.8, 1.9, 2 and 2.1 times the standard deviation of the implied expected returns obtained using the market capitalization weighted portfolio, the risk-efficient portfolio, the maximum diversification portfolio and the equal-risk-contribution portfolio, respectively. Such shrinkage toward the unconditional mean is known to be an important factor of the success of implied expected returns as it reduces the sensitivity of mean-variance portfolios to estimation errors in the return estimates.

A further relevant question is to characterize the dynamics of the differences in returns' predictions between the alternative implied expected return calculations and the standard market capitalization implied portfolio returns.

The slope parameter $b$ is clearly a crucial parameter. As can be seen in (11), when $b=0$, the expected return prediction is identical for all assets. In this case the mean-variance optimization problem is equivalent to a minimum variance optimization problem. In contrast, when $b>0$, differences between the benchmark portfolio weights will lead to different return predictions.

Figure 2 displays the estimated slope coefficients at each rebalancing date for the various implied expected returns approaches. Note that, for all approaches, the months for which the slope coefficient in the implied expected return equation becomes zero almost always coincide. It follows that the timing ability of investing in a implied return portfolio $(b>0)$ or in the minimum variance portfolio $(b=0)$ is, in our sample, not influenced by the choice of a mean-variance efficient portfolio proxy.

We notice large differences in the magnitude of the slope coefficient $b$. The highest average $b$ is observed for the maximum diversification portfolio (average value of 21.17 when $b>0$ ) compared with the market average $b$ which is 7.51 . The difference is due to the fact that the standard deviation of $[\Sigma \mathbf{w}]_{i}$ is 2.75 times smaller (on average, when $b>0$ ) for the maximum diversification portfolio compared with the market portfolio. 

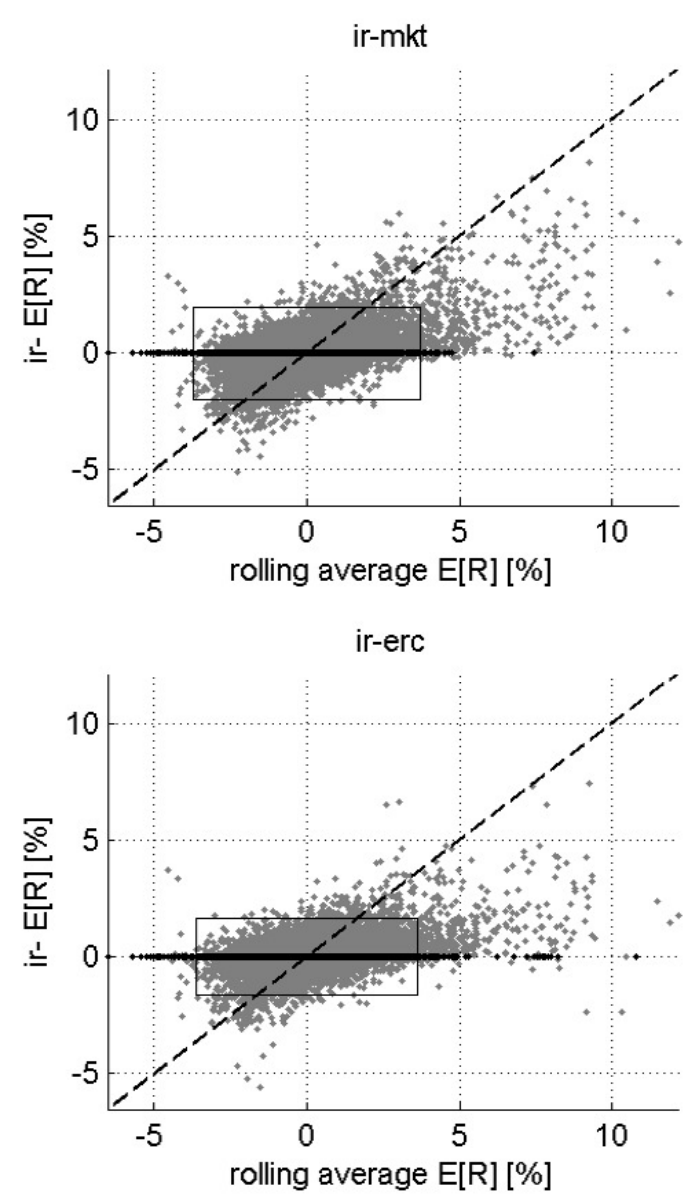
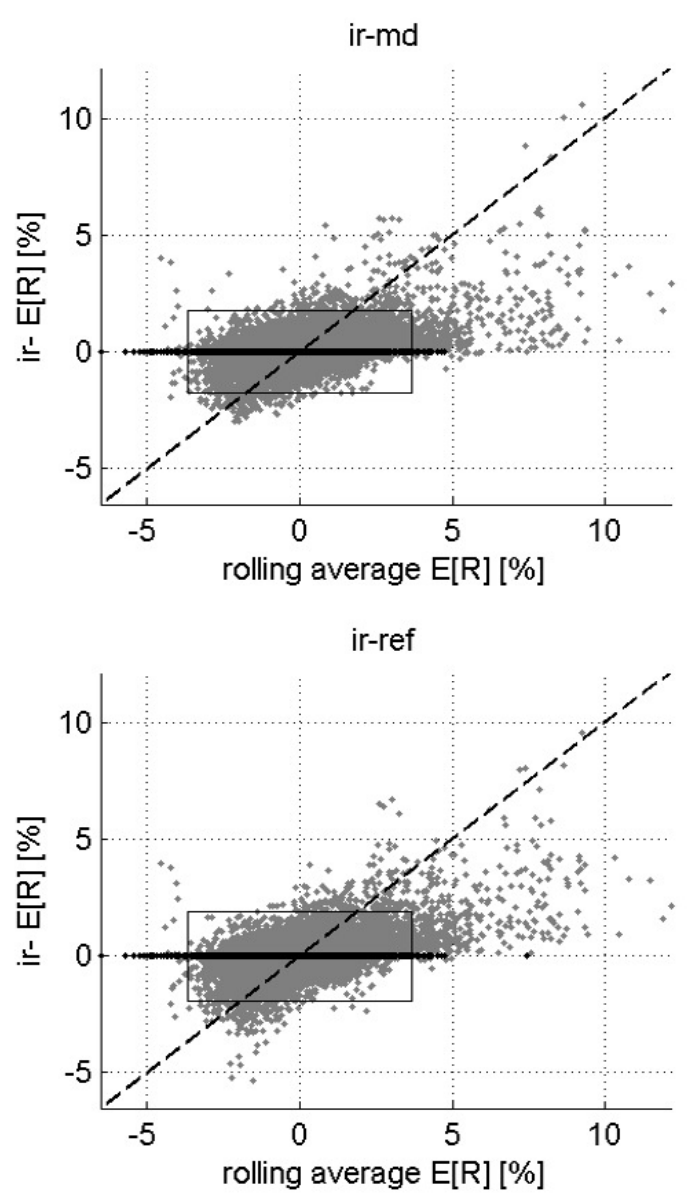

Figure 1: Rolling sample expected return estimate against implied expected returns obtained using the market capitalization weighted portfolio (upper-left), maximum diversification (upper-right), equal-risk-contribution (bottom-left) and risk-efficient (bottom-right) portfolios as mean-variance efficient proxy. Note that expected returns are centered, i.e., the average forecast is subtracted from the expected returns. Gray points report estimates when $b>0$ while black points report estimates when $b=0$. All boxes are centered around zero and their width (resp. height) is six times the standard deviation of the rolling sample estimate (resp. implied return estimate). 

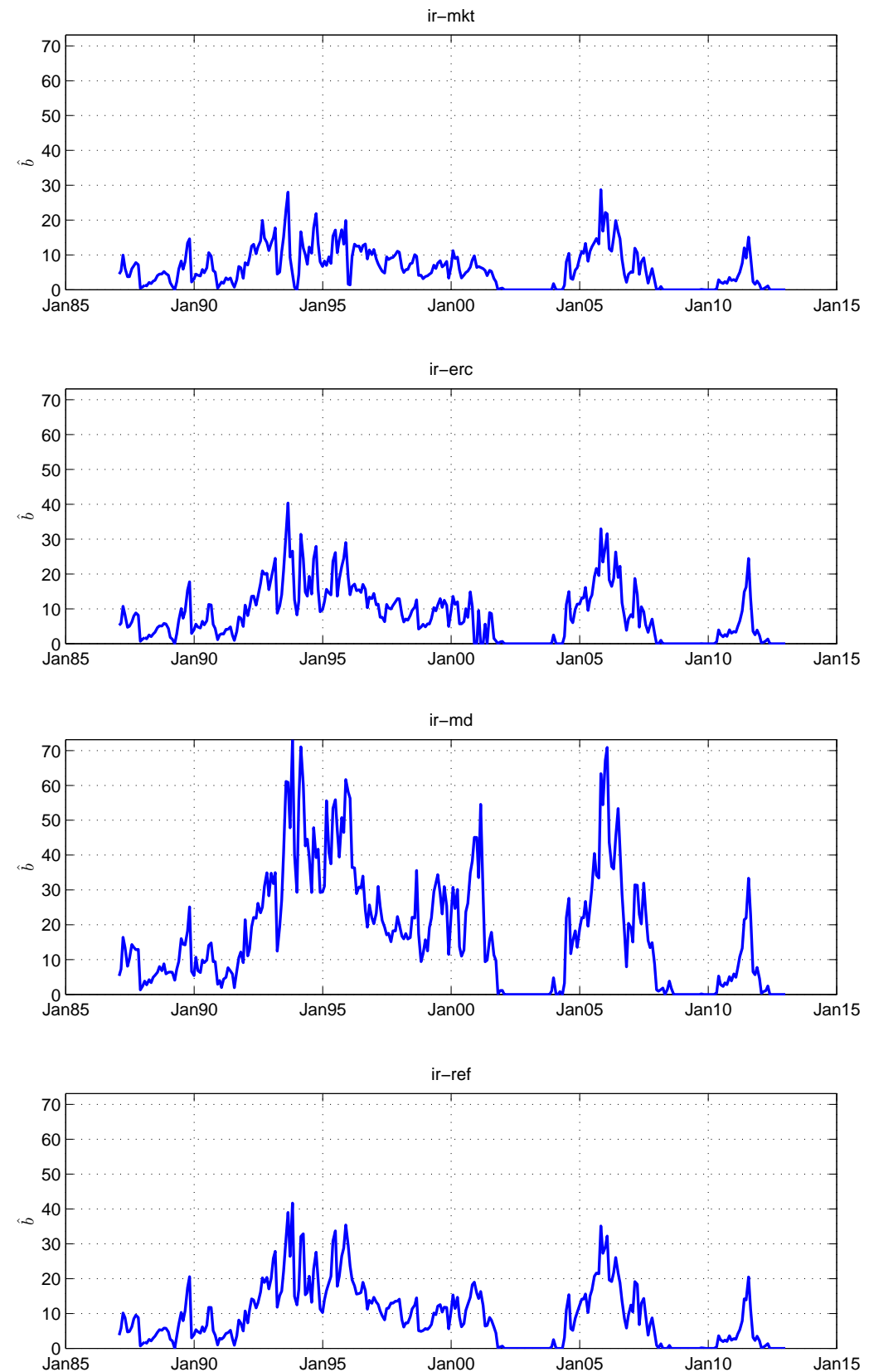

Figure 2: Evolution of the slope coefficient $b$ in (6) for the implied expected returns obtained using the market capitalization weighted (ir-mkt), equal-risk-contribution (ir-erc), maximum diversification (ir-md) and risk-efficient (ir-ref) portfolios as mean-variance efficient proxies. The evaluation period ranges from January 1987 to December 2012 for a total of 312 monthly observations. 


\section{Implied expected returns and mean-variance portfolio allocation}

The previous section documented the relatively high correlation between the different implied return estimates. Still, the choice of implied return calculation method can have a large impact on the performance of the mean-variance investor, as we show below. We focus on three hypothetical mean-variance investors with a high $(\gamma \equiv 3.795)$, medium $(\gamma \equiv 2.7063)$ and low $(\gamma \equiv 0.8773)$ risk aversion, as in Das et al. (2010). ${ }^{4}$

\subsection{Portfolio choices}

The investor has the choice between a large set of portfolios that we categorize as either sensitive or insensitive to the estimated expected returns.

The expected return-insensitive portfolios do not require an expected return forecast. We consider the market capitalization weighted portfolio $\left(\mathbf{w}_{\mathrm{mkt}}\right)$, the smoothed market capitalization weighted portfolio $\left(\mathbf{w}_{\mathrm{smkt}}\right)$, the equally-weighted portfolio $\left(\mathbf{w}_{\mathrm{ew}}\right)$, the equal-risk-contribution portfolio $\left(\mathbf{w}_{\text {erc }}\right)$, the maximum diversification portfolio $\left(\mathbf{w}_{\mathrm{md}}\right)$ and the risk-efficient portfolio $\left(\mathbf{w}_{\text {ref }}\right)$, as described in Section 3. Additionally, we consider the minimum variance portfolio $\left(\mathbf{w}_{\min }\right)$ under a full investment and the gross exposure constraint requiring that the sum of absolute weights should be less or equal to $c \geq 1$ (Fan et al., 2009). The latter is imposed to keep the minimum variance portfolio realistic. We take $c=1$ and $c=1.6$. The case of $c=1$ is equivalent to a long-only portfolio:

$$
\mathcal{C}_{c=1} \equiv\left\{\mathbf{w} \in \mathbb{R}_{+}^{N} \mid \mathbf{w}^{\prime} \iota=1\right\}
$$

As noted by Fan et al. (2009), setting $c=1.6$ corresponds to the typical case of $130 \%$ long positions and $30 \%$ short positions:

$$
\mathcal{C}_{c=1.6} \equiv\left\{\mathbf{w} \in \mathbb{R}^{N}\left|\sum_{i=1}^{N}\right| \mathbf{w}_{i} \mid<1.6, \mathbf{w}^{\prime} \iota=1\right\} .
$$

Overall, we have eight expected return-insensitive portfolios.

The expected return-sensitive portfolios are defined as the solution to the constrained meanvariance utility function (1) with risk aversion level $\gamma$ corresponding to the risk aversion of the investors considered. As such, we obtain for $\gamma=3.795$ a "low risk" portfolio, for $\gamma=2.7063$ a "medium risk' portfolio and for $\gamma=0.8773$ a "high risk portfolio". It is important to observe that, when the mean prediction is identical for all assets, the mean-variance efficient portfolio is the minimum variance portfolio (for all levels of risk aversion $\gamma$ ). When using the implied expected returns in (11), this will happen whenever the estimated slope coefficient $b$ is zero. In line with financial practice, we impose the same constraints as for the minimum variance portfolio. Overall, we have 42 expected return-sensitive portfolios.

\footnotetext{
${ }^{4}$ Note that a direct comparison of statistical accuracy of the implied expected returns is difficult because the expected return parameter itself is not observable and realized returns are extremely noisy. We therefore limit ourselves to the economic evaluation of the implied expected return forecasts by means of an extensive portfolio backtest study.
} 


\subsection{Performance evaluation}

To asses the performance of the various portfolios, we consider: (i) the annualized out-ofsample mean return, (ii) the annualized volatility, (iii) the realized mean-variance utility $\mathrm{RU}_{\gamma}$ for the risk aversion parameter $\gamma$, (iv) the annualized Sharpe ratio and (v) the monthly empirical Valueat-Risk at the $95 \%$ risk level. The realized mean-variance utility is computed as the average out-ofsample mean return minus $\gamma / 2$ times the variance of the out-of-sample portfolio return. Since the out-of-sample mean and variance are unbiased estimates of the unconditional mean and variance, the realized mean-variance utility estimates the expected mean-variance utility function. For the expected return-sensitive portfolios, we also report 12 times the difference between the realized mean-variance utility of any portfolio strategy and the realized utility obtained using the implied returns from the market capitalization weighted portfolio. Simple algebra shows that this measure, denoted $\Phi_{\gamma}$, coincides with the annualized management fee that a mean-variance efficient investor with risk aversion level $\gamma$ would have had to pay (or receive, in case it is negative) to end up with the same utility as when he would have used the implied returns from the market capitalization weighted portfolio. Finally, we also report the portfolio turnover, defined as the average sum of the absolute value of the trades across the $N$ available assets:

$$
\text { Turn } \equiv \frac{1}{T} \sum_{t=1}^{T} \sum_{i=1}^{N}\left|w_{i, t+1}-w_{i, t^{+}}\right|,
$$

in which $w_{i, t+1}$ is the desired portfolio weight at time $t+1$, after rebalancing, and $w_{i, t^{+}}$is the portfolio weight before rebalancing at time $t+1$. The turnover quantity can be interpreted as the average percentage of wealth traded in each period.

\subsection{Results for expected return-insensitive portfolios}

In Table 2 we first analyze the differences in performance of the expected return-insensitive portfolios. To facilitate the interpretation, the lowest and highest value of each performance measure are reported in bold.

Since the annualized average return is around $10 \%$ for all portfolios, the portfolio risk is the distinctive factor. The out-of-sample annualized portfolio volatility and monthly VaR are the lowest for the minimum variance portfolios (around 13\% and -5\%, respectively) and the highest for the (smoothed) market capitalization and equal-weighted portfolios (around 16\% and -8\%, respectively). The Sharpe ratio is the highest (0.403) for the equal-risk-contribution portfolio and the lowest for the market capitalization weighted portfolio (0.304). This is consistent with the growing literature documenting the higher risk adjusted performance of risk optimized portfolios such as the equal-risk-contribution portfolio or the minimum variance portfolio with respect to market capitalization portfolios for this period (see e.g. Baker and Haugen, 2012). The disadvantage of the Sharpe ratio is that it does not reflect the risk aversion of the investor. We find that, in terms of realized utility, the very risk averse investor $(\gamma=3.795)$ is best off with the minimum variance portfolio and worst off with the market capitalization and equally weighted portfolios. The less risk averse investors would have enjoyed the highest utility with the equal-risk-contribution portfolio and the lowest utility with either the market capitalization weighted/equally weighted 
portfolio $(\gamma=2.7063)$ or the minimum variance portfolio with maximum $30 \%$ short sales allowed under the gross exposure constraint $(\gamma=0.8773)$. The turnover of the market capitalization weighted portfolio (9.8\%) is due to changes in the investment universe. The turnover of the equalrisk-contribution (25\%) and risk-efficient portfolio (28\%) is moderate compared with the high turnover of the maximum diversification portfolio $(81.5 \%)$ and the minimum variance portfolio with $30 \%$ short sales allowed (102\%).

\begin{tabular}{lccccccrr}
\hline & $\mathrm{w}_{\text {mkt }}$ & $\mathrm{w}_{\text {smkt }}$ & $\mathrm{w}_{\text {ew }}$ & $\mathrm{w}_{\text {erc }}$ & $\mathrm{w}_{\text {md }}$ & $\mathrm{w}_{\text {ref }}$ & $\mathrm{w}_{\min c=1}$ & $\mathrm{w}_{\min } c=1.6$ \\
\hline Mean & 0.101 & 0.102 & 0.106 & $\mathbf{0 . 1 1 0}$ & 0.104 & 0.109 & 0.104 & $\mathbf{0 . 0 9 7}$ \\
Vol & 0.157 & $\mathbf{0 . 1 5 9}$ & 0.158 & 0.141 & 0.144 & 0.147 & 0.129 & $\mathbf{0 . 1 2 8}$ \\
$\mathrm{RU}_{\gamma=3.795}$ & 0.453 & $\mathbf{0 . 4 4 8}$ & 0.489 & 0.603 & 0.543 & 0.571 & $\mathbf{0 . 6 0 6}$ & 0.549 \\
$\mathrm{RU}_{\gamma=2.7063}$ & 0.565 & $\mathbf{0 . 5 6 3}$ & 0.602 & $\mathbf{0 . 6 9 4}$ & 0.637 & 0.668 & 0.681 & 0.624 \\
$\mathrm{RU}_{\gamma=0.8773}$ & 0.752 & 0.756 & 0.793 & $\mathbf{0 . 8 4 6}$ & 0.795 & 0.832 & 0.808 & $\mathbf{0 . 7 5 0}$ \\
Sharpe & $\mathbf{0 . 3 0 4}$ & 0.305 & 0.334 & $\mathbf{0 . 4 0 3}$ & 0.356 & 0.382 & 0.395 & 0.342 \\
VaR95 & -0.070 & -0.074 & $\mathbf{- 0 . 0 7 6}$ & -0.063 & -0.061 & -0.064 & $\mathbf{- 0 . 0 4 5}$ & -0.048 \\
Turn & 0.098 & $\mathbf{0 . 0 7 7}$ & 0.101 & 0.249 & $\mathbf{0 . 8 1 5}$ & 0.284 & 0.706 & 1.024 \\
\hline
\end{tabular}

Table 2: Out-of-sample results of the expected return-insensitive portfolios. Mean: annualized mean. Vol: annualized volatility. $\mathrm{RU}_{\gamma}$ : realized utility $(\times 100)$ for high $(\gamma=3.795)$, medium $(\gamma=2.7063)$ and low $(\gamma=0.8773)$ risk aversion. Sharpe: annualized Sharpe ratio. VaR95: empirical monthly Value-at-Risk at the 95\% risk level. Turn: average turnover. $\mathbf{w}_{\mathrm{mkt}}$ : market capitalization weighted portfolio; $\mathbf{w}_{\mathrm{smkt}}$ : smoothed market capitalization weighted portfolio; $\mathbf{w}_{\mathrm{ew}}$ : equally-weighted portfolio; $\mathbf{w}_{\mathrm{erc}}$ : equal-risk-contribution portfolio; $\mathbf{w}_{\mathrm{md}}$ : maximum diversification portfolio; $\mathbf{w}_{\text {ref }}$ : risk-efficient portfolio; $\mathbf{w}_{\min }{ }_{c=1}:$ minimum volatility portfolio (long-only constraint); $\mathbf{w}_{\min } c=1.6$ : minimum volatility portfolio (gross exposure constraint). The evaluation period ranges from January 1987 to December 2012 for a total of 312 monthly observations. To facilitate the interpretation, the lowest and highest value of each performance measure are in bold.

\subsection{Results for expected return-sensitive portfolios}

Over the 1987- 2012 period, we thus find that the annualized return of the market capitalization weighted portfolio and the risk-based portfolios are very similar and around $10 \%$. As we show in Table 3, using the three-year rolling sample return forecasts and mean-variance portfolio optimization, it is possible to obtain a higher out-of-sample return at the price of an increased risk. By using implied expected returns, the average return is again around $10 \%$ and the portfolio risk decreases substantially. Based on the Sharpe ratio and the realized utility of the mean-variance investor, all expected return-sensitive portfolios under-perform compared with the expected return-insensitive portfolios in Table 2.

Our main focus is on the impact of the choice of mean-variance efficient portfolio proxy. Here we find the positive result that changing the reference portfolio improves the performance of the expected return-sensitive portfolios. In fact, we find a substantial improvement in the Sharpe ratio and realized utility of mean-variance efficient portfolios computed using the implied expected returns from the equal-risk-contribution portfolio, compared with the standard use of implied expected returns from the market capitalization weighted portfolios. Depending on the risk aversion level an investor has, he would be willing to pay between $1.7 \%$ and $4.2 \%$ additional management fee to switch from mean-variance investing using implied expected returns from the 
market capitalization weighted portfolio to mean-variance investing using implied expected return from the equal-risk-contribution portfolio.

The superiority of the equal-risk-contribution portfolio implied expected excess returns does not generalize to all risk optimized portfolios considered. The performance of the implied return using smoothed market capitalization weights or equal weights is similar to the implied expected returns from the market portfolio. Especially the implied expected returns from the maximum diversification portfolio are for this sample less informative for the mean-variance investor, resulting in Sharpe ratios and utilities that are lower than when using expected returns implied by the market portfolio. The results for the implied expected returns from the risk-efficient portfolio are mixed. 


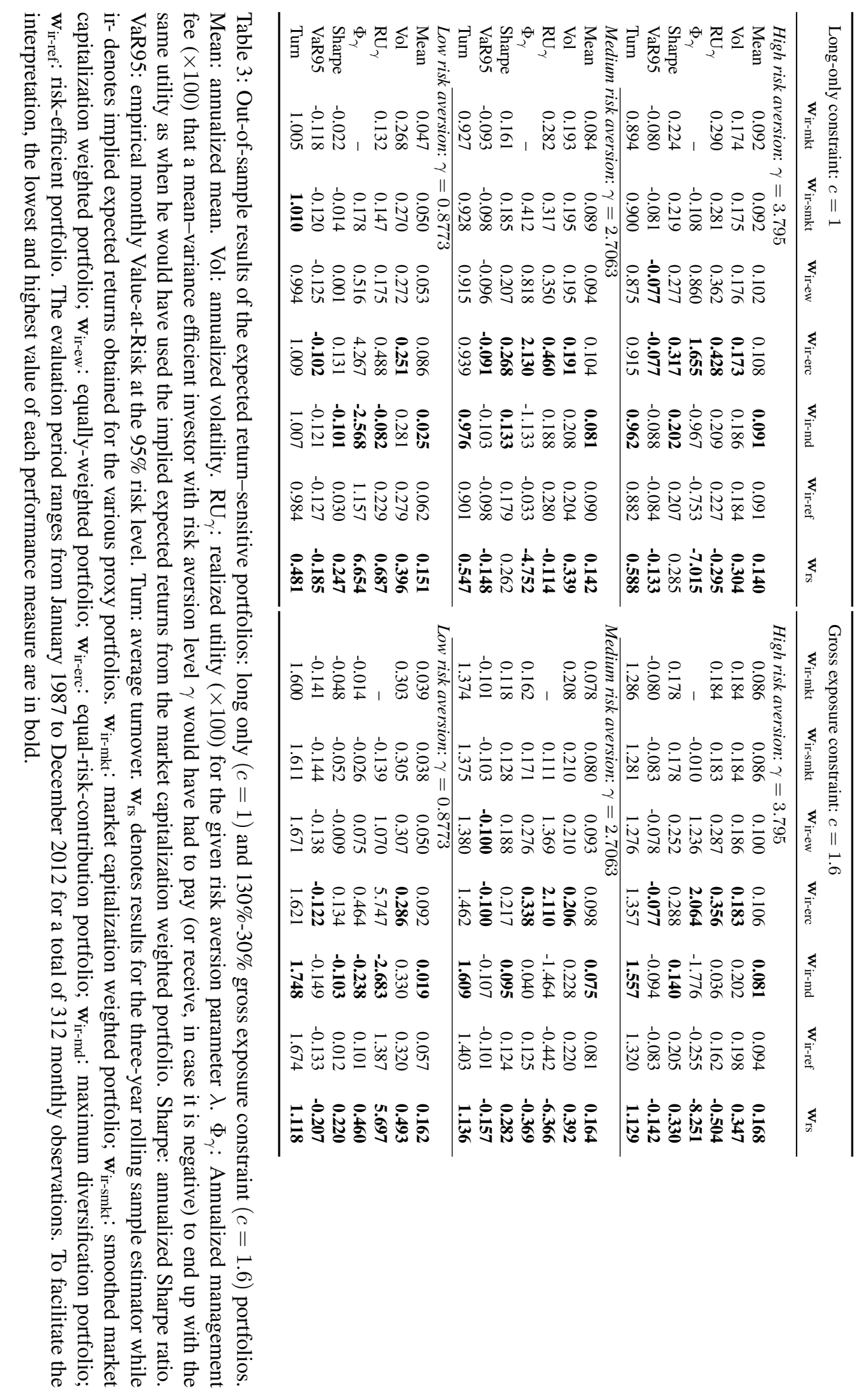




\subsection{Robustness analysis}

We have carried out several robustness analyses. In particular, we investigated the impact on our results to the choice of the estimation window and the persistence parameter in the covariance estimation. Overall, the main results are unaffected. Details are available from the authors upon request.

\section{Conclusion}

We study the performance of mean-variance portfolios constructed using implied expected returns obtained as the least squares fit of a first-step mean estimate (the historical average) on the regressors implied by the fundamental relation between the expected returns, covariance matrix and the corresponding set of mean-variance efficient portfolios. Our motivation is that, even though traditionally the market capitalization weighted portfolio dominates in terms of reference portfolio from which the implied expected returns are derived, there is growing empirical evidence that risk-based portfolios may be a better proxy for a mean-variance efficient portfolio. In fact, in his important review on risk-based asset allocation, Lee (2011) concludes that if risk-based allocation approaches outperform the market portfolio, it must be that the implied expected returns are a better set of return forecasts than the market's implied expected returns. We investigate empirically this prediction for the universe of S\&P 100 stocks over a period ranging from January 1987 to December 2012 and find that substantial improvement in portfolio performance can be achieved. In particular, we estimate that, over the January 1987-December 2012, a mean-variance efficient investor would have been willing to pay between a $1.7 \%$ and $4.2 \%$ management fee to switch from mean-variance investing using implied expected returns from the market capitalization weighted portfolio to mean-variance investing using the implied expected returns from the equal-risk-contribution portfolio.

Our research on risk-based portfolio allocation and implied expected returns can be extended in several ways. In our empirical analysis, we documented that there is no general superiority of implied expected returns from risk-based portfolios. The validity of using the market capitalization weighted portfolio or the different risk-based portfolios as a mean-variance efficient portfolio proxy depends on the market regime. We are currently investigating the gains of dynamically switching between implied expected return calculation methods. In addition, we are investigating portfolio allocation strategies that account for the joint uncertainty in the mean and covariance matrix, as an extension of the recent work by Levy and Roll (2010).

\section{References}

Amenc, N., Goltz, F., Martellini, L., Retkowsky, P., 2011. Efficient indexation: An alternative to cap-weighted indices. Journal of Investment Management 9 (4), 1-23.

Arnott, R., Hsu, J., Moore, P., 2005. Fundamental indexation. Financial Analysts Journal 61, 83-89.

Baker, N. L., Haugen, R. A., April 2012. Low risk stocks outperform within all observable markets of the world. Working paper.

Best, M. J., Grauer, R. R., 1985. Capital asset pricing compatible with observed market value weights. Journal of Finance 40 (5), 85-103. 
Black, F., Litterman, R., September-October 1992. Global portfolio optimization. Financial Analyst Journal 48 (5), 28-43.

Campbel, J. Y., Thompson, S. B., 2007. Predicting excess stock returns out of sample: Can anything beat the historical average? Review of Financial Studies 21, 1509-1531.

Chen, C., Rong, C., Bassett, G. W., 2007. Fundamental indexation via smoothed cap weights. Journal of Banking and Finance 31, 3486-3502.

Choueifaty, Y., Coignard, Y., 2008. Toward maximum diversification. Journal of Portfolio Management 35 (1), 40-51.

Choueifaty, Y., Froidure, T., Reynier, J., 2011. Properties of the most diversified portfolio. Working paper.

Das, S., Markowitz, H., Scheid, J., Statman, M., 2010. Portfolio optimization with mental accounts. Journal of Financial and Quantitative Analysis 45, 311-334.

DeMiguel, V., Garlappi, L., Uppal, R., 2009. Optimal versus naive diversification: How inefficient is the 1/n portfolio strategy. The Review of Financial Studies 22 (5), 1915-1953.

Fan, J., Zhang, J., Yu, K., March 2009. Asset allocation and risk assessment with gross exposure constraints for vast portfolios.

Flood, C., 2013. Big spike in "smart beta" investments. Financial Times (17-06-2013).

Jorion, P., 1986. Bayes-stein estimation for portfolio analysis. Journal of Financial and Quantitative Analysis 21 (3), 279-292.

Ledoit, O., Wolf, M., 2003. Improved estimation of the covariance matrix of stock returns with an application to portfolio selection. Journal of Empirical Finance 10, 603-621.

Lee, W., 2011. Risk-based asset allocation: A new answer to an old question? Journal of Portfolio Management, Summer 2011, 11-28.

Levy, M., Roll, R., 2010. The market portfolio may be Mean-Variance efficient after all. The Review of Financial Studies 23 (6), 2464-2491.

Maillard, S., Roncalli, T., Teiletche, J., 2010. The properties of equally weighted risk contribution portfolios. Journal of Portfolio Management 36 (4), 60-70.

Martellini, L., 2008. Towards the design of better equity benchmarks. Journal of Portfolio Management 34, Summer, 34-41.

Merton, R. C., 1980. On estimating the expected return on the market. Journal of Financial Economics 8, 323-361.

Meucci, A., 2001. Common pitfalls in mean-variance asset allocation. Wilmott Magazine.

Meucci, A., 2004. Broadening horizons. Risk 17 (12), 98-101.

Ni, X., Malevergne, Y., Sornette, D., Woehrmann, P., 2011. Robust reverse engineering of cross-sectional returns and improved portfolio allocation performance using the CAPM. Journal of Portfolio Management 37 (4), 76-85.

RiskMetrics Group, 1996. RiskMetrics Technical Document. J. P. Morgan/Reuters, 4th Edition.

Treynor, J. L., 2005. Why market-valuation-indifferent-indexing works. Financial Analysts Journal 61 (5), 65-69.

Welch, I., Goyal, A., 2007. A comprehensive look at the empirical performance of equity premium prediction. Review of Financial Studies 21, 1455-1508. 